\title{
LES EVOLUTIONS RECENTES DE LA DECENTRALISATION AU SENEGAL
}

\author{
Par Maimouna A $S Y^{1}$
}

\section{Introduction}

Dans un pays moderne, la centralisation absolue c'est-à-dire la concentration de l'ensemble des compétences entre les mains du pouvoir central, se heurte à une difficulté voire une impossibilité manifeste en raison notamment de la diversité des tâches qui incombent à l'Etat. Dès lors, il est nécessaire que les décisions d'intérêt local soient prises au niveau local afin d'éviter toute paralysie des organes centraux. A cet effet, il s'est agi de mettre en place deux techniques de gestion administrative pour désengorger le pouvoir central. Il s'agit de la déconcentration et de la décentralisation. Raogo Antoine SAWADOGO, dans son ouvrage intitulé « l'Etat africain face à la décentralisation » asserte que la décentralisation est présentée comme le droit pour les collectivités territoriales ou locales de s'administrer librement et de gérer des affaires propres en vue de promouvoir le développement à la base et de renforcer la gouvernance locale. C'est donc l'axe fondamental d'impulsion du développement et de la démocratie ${ }^{2}$. Ainsi, nous nous accorderons avec Annie GRUBER, qui dans son ouvrage intitulé « la décentralisation et les institutions administratives » souligne que la différence essentielle entre la déconcentration et la décentralisation est d'ordre politique et tient au statut des organes qui bénéficient de la redistribution du pouvoir : dans le cadre de la déconcentration, ces organes sont le reflet fidèle et l'instrument docile du pouvoir central c'est-à-dire une technique de commandement dans laquelle l'agent déconcentré est subordonné à l'autorité de l'Etat; dans celui de la décentralisation, ils ont leur identité propre et jouissent de plus d'autonomie ${ }^{3}$. L'autonomie qui est une caractéristique essentielle des collectivités locales a été consacrée par la constitution sénégalaise à travers l'affirmation du principe de la libre administration des collectivités locales, et qui en son article 102 stipule que les collectivités locales constituent le cadre institutionnel de la participation des citoyens à la gestion des affaires publiques. Elles s'administrent librement par des assemblées élues ${ }^{4}$.

Ainsi, «Le Sénégal depuis son accession à la souveraineté internationale a opté pour une politique de décentralisation prudente, progressive et irréversible $»^{5}$. Partant, un bref rappel de la décentralisation semble indispensable. En effet, avant son accession à la souve-

1 DOCTORANTE EN DROIT PUBLIC, UGB, SAINT LOUIS.

2 Raogo Antoine SAWADOGO, L'Etat africain face à la décentralisation, Editions Karthala, 2001.

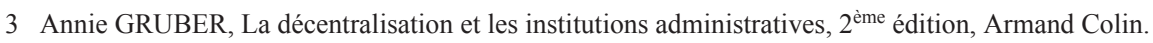

4 Article 102 de la constitution sénégalaise du 22 janvier 2001.

5 Exposé des motifs du nouveau code général des collectivités locales. 
raineté internationale, l'on a assisté au Sénégal à la création des communes de Saint-Louis et de Gorée en 1872. L'année 1880 fut marquée par la création de la commune de Rufisque et 1887 par celle de la commune de Dakar. En 1955, la loi municipale élargit la communalisation avec la mise sur pied des communes de statut juridique différent. L'année 1960 a été marquée par l'élargissement du statut de commune de plein exercice à l'ensemble des communes. En 1964, un statut spécial fut conféré à la région de Dakar, la commune région du Cap Vert est administrée par le gouverneur de la région, puis par un administrateur de la commune à partir de 1979. L'année 1966 correspond à la promulgation de la loi nº6-64 du 30 juin 1966 portant code de l'administration communale. En 1972, l'on créa une deuxième catégorie de collectivité locale à savoir les communautés rurales. En 1983, Dakar reprend son statut de droit commun (commune de plein exercice). L'année 1990 coïncide avec la suppression des communes à statut spécial qui deviennent des communes de plein exercice, mais également au transfert des pouvoirs d'ordonnateur du budget des sous-préfets aux présidents de conseils ruraux. En 1996, la région fut érigée en collectivité locale et l'on assiste en même temps au transfert de neuf de domaines de compétences aux différentes collectivités locales que sont les régions, les communes et les communautés rurales. A cela s'ajoute la création à la même année des communes d'arrondissement et la substitution du contrôle a priori au contrôle de légalité. Enfin, l'année 2013 correspond au lancement officiel de « l'acte 3 » de la politique de décentralisation au Sénégal. Fort de ce constat, l'on peut affirmer que la décentralisation au Sénégal est marquée par plusieurs étapes. Toutefois, si d'aucuns classent la nouvelle réforme comme étant l'acte 3 c'est dû au fait qu'ils considèrent la réforme de 1972 correspondant à la création d'une nouvelle collectivité locale en l'occurrence la communauté rurale comme étant l'acte 1. Quant à la réforme de 1996, elle est considérée comme l'acte 2 au regard des innovations importantes qu'elle a engendrées, marquant ainsi une étape décisive au processus de décentralisation au Sénégal. Néanmoins, malgré la volonté des autorités étatiques de faire des collectivités locales de véritables pôles de développement, la décentralisation au Sénégal s'avère présenter des lacunes voire des limites avec notamment le défaut de ressources humaines et financières de ces dites collectivités. De ce fait, le pouvoir en place, après son installation, a pris l'initiative d'aller vers une nouvelle réforme pour lutter contre les inégalités qui existent au sein des collectivités locales au Sénégal. A cet effet, il a été mis sur pied un comité de pilotage pour réfléchir sur la conception de ce qu'il dénomme l'acte 3 de la décentralisation pour une meilleure prise en compte des intérêts locaux en évitant toute sorte de discrimination entre collectivités locales. A cette occasion, suite aux réflexions menées par le comité de pilotage de «l'acte 3 » de la décentralisation, il s'est agi de supprimer la région en tant que collectivité locale et d'ériger le département en une nouvelle collectivité locale. A cela s'ajoute la suppression des communautés rurales et des communes d'arrondissement en procédant à cet effet à la communalisation intégrale, entre autres innovations. En conséquence, on a assisté à l'élargissement des compétences de la commune et l'octroi au département de nouvelles compétences. Telles sont entre autres les innovations majeures de la première phase « de l'acte 3 » de la décentralisation. Cette première phase est mise en évidence par la loi 2013-10 du 28 
décembre 2013 portant code général des collectivités locales (CGCL). On entend ainsi par innovations toute forme de changement, de nouveauté par rapport à une situation préexistante. Par ailleurs, Gérard CORNU définit le « code » comme étant un corps de textes englobant selon un plan systématique l'ensemble des règles relatives à une matière et issu, soit de travaux législatifs, soit d'une élaboration réglementaire, soit d'une codification formelle de textes préexistants reclassés selon leur origine ${ }^{6}$. Quant au terme «collectivité locale », l'auteur précité le définit comme un ensemble d'habitants d'une même partie du territoire ayant des intérêts communs gérés par des organes administratifs qui lui sont propres, créé comme tel par la constitution ou par la loi ${ }^{7}$. Djibril Diop, dans son ouvrage intitulé «Décentralisation et gouvernance locale au Sénégal : quelle pertinence pour le développement local? », définit « les collectivités locales comme une communauté sociale infra-étatique, qui, ayant pour assise une fraction directe du territoire national et pour lien une identité collective locale dotée de la personnalité morale lui permettant de gérer ses propres affaires ». De ces définitions, il apparaît des caractéristiques essentielles de la notion de collectivité locale à savoir : l'octroi de la personnalité juridique aux organes locaux qui se manifeste par l'élection de ces dits organes par la population de la collectivité mais aussi la notion d'affaires locales. En effet, ce sujet portant sur les innovations introduites par le code général des collectivités locales au Sénégal renferme un intérêt certain en ce sens qu'il nous permettra d'exposer les innovations apportées par « l'acte 3 » notamment la première phase et de juger également de sa pertinence et éventuellement de ses lacunes. Dès lors plusieurs questions pourraient être soulevées : quelles sont les différentes innovations apportées par le CGCL? Cette nouvelle réforme a-t-elle pris en considération tous les problèmes rencontrés au sein des collectivités locales? Quels sont ses effets sur les plans institutionnel et fonctionnel? Cette réforme est-elle réellement pertinente? En effet, pour mieux cerner l'ensemble des interrogations auxquelles suscite ce sujet, nous nous poserons la question de savoir : quelle appréciation pouvons-nous faire des innovations introduites par le code général des collectivités locales au Sénégal? En effet, tel qu'évoqué au niveau de l'exposé des motifs du code général des collectivités locales, le contexte et la faiblesse des politiques et stratégies de développement appliquées jusque-là, nécessitent, en conséquence, d'initier des alternatives susceptibles de corriger les déficiences et de produire simultanément des progrès significatifs à l'échelle nationale et un développement local harmonieux ${ }^{8}$. Partant, les autorités, afin de corriger les disparités notées entre les collectivités locales et dans un souci de promouvoir une gestion de proximité, sont allées dans le sens d'une réforme du cadre institutionnel des collectivités locales. Pour ce faire, elles (autorités) ont procédé à la suppression des communautés rurales et des communes d'arrondissement en les élevant à cet effet au rang de communes. De plus, la région en tant que collectivité locale a été éliminée

6 Gérard CORNU, Vocabulaire Juridique, $7^{\text {ème }}$ édition revue et augmentée, PUF, page 151, 1998.

7 Idem, page 156.

8 Exposé des motifs de la loi 2013-10 du 28 décembre 2013 portant code général des collectivités locales. 
et en lieu et place l'on a conféré au département le statut de collectivité locale à l'instar des communes. De ce fait, le Sénégal qui renfermait jusque-là trois ordres de collectivités locales, se retrouve avec deux que sont le département et la commune. Par ailleurs, la ville s'est également vu octroyer un nouveau statut. Du reste, il va sans dire que, les innovations du point de vue institutionnel auront dans une certaine mesure certaines répercussions sur le fonctionnement des collectivités locales. En conséquence, un changement sera noté dans le cadre du transfert de compétences. Ainsi, un vaste domaine de compétences sera dévolu au département et les communes verront leurs compétences considérablement élargies. Aussi, sur le plan financier, les nouvelles communes bénéficieront de nouvelles ressources financières mais aussi une partie des fonds des communes sera transférée aux villes afin d'assurer leur fonctionnement. A ces innovations sur le plan fonctionnel, s'ajoute également la revalorisation de la participation citoyenne à travers sa consécration formelle. En effet, la participation citoyenne qui était évoquée au niveau des dispositions générales de l'ancien code des collectivités locales, fait désormais l'objet d'un intitulé au Titre premier du CGCL. D’un autre côté, notons que le CGCL n'en est évidemment qu'à ses débuts, par conséquent il est très tôt d'établir son bilan. Cependant, l'on peut tout de même se permettre d'apprécier ses dispositions par rapport à celles de l'ancien code des collectivités locales mais également au regard des attentes ou des espoirs suscités. En d'autres termes, l'élaboration du CGCL a-t-elle apporté des solutions aux maux dont souffrait pour l'essentiel le processus de la décentralisation au Sénégal? En effet, à la lecture des dispositions du code général des collectivités locales, la réponse semble négative en ce sens que le nouveau code reprend essentiellement les principes régis par l'ancien code. Aussi, autre facteur bloquant la mise en œuvre de la réforme, c'est la mise en place tardive des textes réglementaires. En outre, il est également constaté que les autorités n'ont pas pris en compte le principal problème dont souffre la bonne marche du processus de décentralisation au Sénégal à savoir la problématique du financement des collectivités locales et la qualité des ressources humaines, notamment. De ce fait, relativement à ce qui précède, l'approche sera bâtie suivant deux axes d'analyse. Dans cette perspective, nous essayerons en premier lieu de mettre en exergue le caractère notoire des innovations apportées par le CGCL (I) et en second lieu, nous tenterons de démontrer que ces innovations sont relatives (II).

\section{I) Les innovations notoires du code général des collectivités locales du Sénégal}

Des innovations apportées par le code général des collectivités locales, apparait l'octroi du statut de collectivité locale au département et la suppression de la région en tant que collectivité locale. Aussi, les communautés rurales et les communes d'arrondissement sont élevées au rang de communes d'où l'expression de communalisation intégrale. A cela s'ajoute l'octroi d'un nouveau statut à la ville mais aussi l'on assiste à la revalorisation de la participation citoyenne. Par ailleurs, cette nouvelle architecture des collectivités locales conduira à une nouvelle répartition des compétences transférées et une certaine modification des ressources financières sera également notée. Ce qui nous amènera à étudier en premier lieu les 
innovations d'ordre institutionnel (A) et en second lieu nous mettrons en exergue les innovations du point de vue fonctionnel (B).

\section{A) Sur le plan institutionnel}

La conception du code général des collectivités locales a fait naître deux ordres de collectivités locales au Sénégal. En effet si la réforme de 1996 de la politique de décentralisation au Sénégal avait amené les ordres de collectivités locales à trois à savoir la région, la commune et la communauté rurale, celle de 2013 réduit le nombre à deux en l'occurrence le département et la commune. Ainsi, le département se voit ériger en collectivité locale à la place de la région et les communautés rurales et les communes d'arrondissement sont élevées au rang de communes à travers la communalisation intégrale.

- En effet, jusqu'à la réforme de 1996, le département n'était qu'une simple circonscription administrative. Autrement-dit, le département n'était qu'un organe déconcentré avec à sa tête le préfet comme représentant de l'Etat au niveau communal. Cependant, avec la nouvelle réforme initiée par les autorités, non seulement le département conserve son ancien statut (c'est-à-dire celui de circonscription administrative) mais aussi il revêt un nouveau statut à savoir celui de collectivité locale à la place de la région. Il sera à cet effet considéré comme échelon de gestion des solidarités sociales et du développement territorial $^{9}$. Partant, le département bénéficiera bien entendu de la personnalité juridique qui impliquera d'ailleurs la gestion de ses propres affaires par des organes propres issus de ladite collectivité par le biais de l'élection. En atteste l'article premier du CGCL qui dispose que « les collectivités locales sont dotées de la personnalité morale et de l'autonomie financière. Elles s'administrent librement par des conseils élus au suffrage universel $»^{10}$. Ainsi, on assiste à l'avènement d'une nouvelle organisation et d'un nouveau fonctionnement du département en tant que collectivité locale. Partant, l'article 20 du CGCL dispose que le département est une collectivité locale, personne morale de droit public. Il est administré par un conseil départemental élu au suffrage universel. Le conseil départemental par ses délibérations, le président du conseil départemental par l'instruction des affaires et l'exécution des délibérations concourent à l'administration du département ${ }^{11}$. Donc, le conseil départemental est l'instance délibérante du département et est composé de conseillères et de conseillers élus pour un mandat de cinq ans. Soulignons qu'à cet effet, l'on a assisté lors des dernières élections locales à une exigence de la parité sur les listes électorales. Autrement-dit, chaque liste devait contenir un nombre égal d'hommes et de femmes pour être recevable. Par ailleurs, le conseil départemental élit à son sein un bureau composé d'un président, d'un premier vice-président,

9 Cheikhou Oumar SOW, «L'acte 3 de la décentralisation» in Afrique démocratie, $\mathrm{n}^{\circ} 22$ Novembre 2013, page 9.

10 Article premier alinéa 2 du Code Général Collectivités Locales de 2013.

11 Article 20 du code général des collectivités locales de 2013. 
d'un second vice-président, et de deux secrétaires. A côté de l'organe délibérant, il y'a le président du conseil qui est l'organe exécutif du département élu également pour cinq ans. Il prépare et exécute les délibérations de l'organe délibérant. Il est l'ordonnateur des dépenses et prescrit l'exécution des recettes du département sous réserve des dispositions particulières du code général des impôts relatives au recouvrement des recettes fiscales des collectivités locales. En tant que chef des services décentralisés du département, le président du conseil départemental peut sous sa surveillance et sa responsabilité, donner délégation de signature aux membres du bureau. En outre, il nomme le secrétaire général du département après avis consultatif du représentant de l'Etat, parmi les agents et fonctionnaire de la hiérarchie $\mathrm{A}$ de la fonction publique ou de niveau équivalent dans les conditions précisées par décret. A cet effet, il peut aussi déléguer sa signature au secrétaire général du département. Ce dernier assiste aux réunions par voix consultative. Dans l'exercice de ses fonctions, le président du conseil peut également disposer des services déconcentrés de l'Etat dans le cadre d'une convention signée avec le représentant de l'Etat, précisant les conditions de prise en charge par le département de ces missions.

Le conseil départemental a son siège au chef-lieu du département. Après l'élection de son bureau, le conseil départemental forme ses commissions, désigne ses membres ou ses délégués pour les représenter au niveau des organismes extérieurs. Les commissions sont au nombre de quatre (4) et sont établies comme suit :

- Commission des affaires administratives, juridiques et du règlement intérieur;

- Commission de l'éducation, de la santé et de la population, des affaires sociales et culturelles, de la jeunesse et des sports;

- Commission des finances, du plan et du développement économique;

- Commission de l'environnement, de l'aménagement du territoire, des domaines, de l'urbanisme et de l'habitat.

Il est fait mention dans le CGCL que toute autre commission peut être créée ou dissoute par délibération du conseil départemental, sur demande de son président ou sur proposition d'au moins un tiers (1/3) des membres du conseil départemental. Outre les commissions, le conseil départemental désigne parmi ses membres des délégués appelés à siéger au sein d'organismes extérieurs dans les cas et conditions prévus par les textes régissant ces organismes. La fixation par les textes précités de la durée des fonctions assignées à ces membres ou délégués ne fait pas obstacle à ce qu'il puisse être procédé, à tout moment et pour le reste de cette durée, à leur remplacement. Par ailleurs, le conseil départemental se réunit une fois par trimestre, en session ordinaire. Chaque session dure quinze (15) jours au plus sauf la session budgétaire qui peut aller jusqu'à un mois. Le conseil se réunit aussi en session extraordinaire pour une durée qui ne peut dépasser trois (3) jours, sur un ordre du jour déterminé à la demande du président, du tiers des membres du conseil départemental (un même conseiller départemental ne peut être signataire de plus d'une demande de réunion par année) et du représentant de l'Etat. Les séances du conseil départemental se tiennent publiquement sauf si le conseil en décide autrement à la majorité absolue des 
membres présents ou représentés. Les délibérations sont prises à la majorité simple. Le vote a lieu au scrutin public mais il peut être tenu secret à la demande du quart (1/4) des membres présents. Lorsqu'il y'a égalité de voix, celle du président est prépondérante. En outre, le conseil départemental ne peut délibérer que si la majorité absolue de ses membres en exercice est présente à l'ouverture de la session. Si toutefois ce quorum n'est pas atteint, la réunion est convoquée de plein droit huit (8) jours plus tard. En l'espèce, les délibérations ne sont valables que si, au moins, le quart des membres du conseil est présent. De plus, le président du conseil départemental transmet aux conseillers départementaux un rapport sur chacune des affaires qui doivent leur être soumise au moins quinze jours avant la réunion du conseil. Il présente aussi chaque année à la fin de sa gestion un rapport spécial au conseil départemental. Soulignons que les fonctions de président, de membre du bureau, de conseiller départemental, de président et de membre de délégation spéciale, donnent lieu sur le budget du département au paiement d'indemnités ou remboursement de frais que nécessite l'exécution des mandats qui leur sont confiés. Notons par ailleurs que la présence du représentant de l'Etat ou de son délégué dûment mandaté lors des séances du conseil est de plein droit. Néanmoins, le représentant de l'Etat ne peut ni présider les séances ni participer au vote. Par ailleurs, en dehors de la départementalisation, l'autre innovation phare du CGCL se trouve être la communalisation intégrale.

- Ainsi, à travers sa vision de la communalisation intégrale, le législateur entend dissiper les clivages ruraux et urbains. Il s'agira également d'après le chef de l'Etat d'améliorer la gouvernance locale et de réduire le gap qui existe entre les zones rurales et les zones urbaines. Ainsi, la commune est considérée comme échelon de proximité et des services de base. A cet effet, a-t-on procédé à l'élévation des communautés rurales au rang de communes et à l'érection des communes d'arrondissement en communes.

En effet, la plupart des communautés rurales et des communes d'arrondissement devenues désormais communes sont caractérisées par la rareté des ressources. Le caractère dérisoire de leurs ressources financières ne permet guère la réalisation d'infrastructures utiles pour impulser leur développement. Ainsi, dans un souci de vouloir corriger cet état de fait, l'Etat sénégalais s'est lancé dans une dynamique de communalisation intégrale en conférant le statut de communes à toutes les communautés rurales et communes d'arrondissement. Dans cette même perspective, le ministre de la gouvernance locale, du développement et de l'aménagement du territoire affirme dans la revue « Afrique Démocratie » que la communalisation intégrale en plus du fait qu'elle permet de simplifier l'architecture territoriale et de dissiper les clivages urbains-ruraux, offre plusieurs avantages à savoir le renforcement des ressources humaines, techniques et financières des nouvelles communes; la possibilité pour elles de bénéficier davantage de la coopération décentralisée et l'amélioration de l'accès à certains services sociaux de base ${ }^{12}$. De ce fait, la communauté rurale qui était jusque-là dirigée par le président du conseil rural (organe exécutif) et le conseil rural (organe délibérant)

12 Interview de Ministre de l'aménagement du territoire et des collectivités locales sur l'acte 3 de la décentralisation, Afrique Démocratie, Novembre 2013, p 19. 
va revêtir un nouveau statut à savoir celui de commune. En conséquence, les nouveaux dirigeants seront dénommés maire (organe exécutif) et conseillers municipaux (organe délibérant). Ainsi, ces derniers sont élus au suffrage universel pour une durée de cinq (5) ans. Le bureau du conseil municipal est composé du maire et de ses adjoints. L'élection du maire et de ses adjoints a lieu au scrutin secret et à la majorité absolue. Les membres du bureau en raison des responsabilités qui leur sont dévolues, doivent savoir lire et écrire. Par ailleurs, après le maire et les adjoints dans l'ordre de leur élection, les conseillers municipaux prennent rang dans l'ordre du tableau. Cet ordre est déterminé par la date la plus ancienne des élections intervenues depuis le dernier renouvellement intégral du conseil municipal mais également entre conseillers élus le même jour, par la priorité d'âge. Il est fait mention dans le CGCL que le maire et les adjoints doivent résider dans la commune ou en être obligatoirement contribuables. Notons également qu'un poste d'adjoint spécial peut être institué par délibération motivée du conseil municipal lorsqu'un obstacle quelconque ou l'éloignement rend difficiles, dangereuses ou momentanément impossibles les communications entre le chef-lieu et une fraction de la commune. Cet adjoint spécial est élu parmi les conseillers résidant dans cette fraction de la commune et, à défaut ou s'il est empêché, parmi les habitants de cette fraction de commune. A cet effet, il joue le rôle d'officier d'état civil et peut être chargé d'exécuter les lois et règlements de police dans cette fraction de commune. Tout comme le département, les fonctions de maire, de membres du bureau, de conseiller municipal, de président et de membre de délégation spéciale, donnent lieu au paiement d'indemnités ou remboursement des frais que nécessite l'exécution des mandats qui leur sont confiés.

De plus, les communes d'arrondissement devenues communes bénéficient d'un nouveau fonctionnement à l'instar des anciennes communautés rurales devenues aussi communes. A cet effet, leur fonctionnement tourne essentiellement autour des activités du conseil municipal à savoir les réunions et les délibérations.

- Sous un autre registre, la ville était considérée dans l'ancien code comme un espace qui regroupe les différentes communes d'arrondissement et elle n'était étudiée que sous l'angle de ses rapports avec la commune d'arrondissement à travers la loi 96-09 du 22 mars 1996 portant organisation administrative et financière de la commune d'arrondissement et ses rapports avec la ville. Cependant, avec le CGCL, la ville devient un cadre de mutualisation des communes établies dans la limite de son territoire. Aussi, contrairement au code des collectivités locales (CCL) de 1996, le code général des collectivités locales de 2013 consacre un certain nombre de dispositions relatives à la ville. Ainsi, l'article 167 du CGCL stipule qu'une ville peut être instituée, par décret, pour mutualiser les compétences de plusieurs communes qui présentent une homogénéité territoriale. Ce décret détermine le nom de la ville, en situe le chef-lieu et en fixe les limites qui sont celles des communes constitutives.

La ville est composée du conseil de la ville, du maire de la ville, des adjoints au maire et du secrétaire général. Le conseil de la ville, organe délibérant, est composé de conseillères et de conseillers désignés pour cinq ans. Contrairement aux conseillers départementaux et mu- 
nicipaux qui sont élus au suffrage universel direct, les conseillers de la ville sont désignés par les communes constitutives. Les conseillers prennent rang dans l'ordre du tableau qui est déterminé comme suit : d'une part, par la date la plus ancienne des élections intervenues depuis le dernier renouvellement intégral du conseil de la ville, et, d'autre part, entre conseillers élus le même jour, par la priorité d'âge. L'élection du maire de la ville qui suit le renouvellement général du conseil a lieu huit jours après celle des maires des communes constituant la ville. Ainsi, le conseil de la ville élit le maire et ses adjoints parmi ses membres sachant lire et écrire. La détermination du nombre des adjoints du maire de la ville correspond aux mêmes règles que celles qui président la détermination du nombre d'adjoints du maire de la commune. Notons que les fonctions de maire de ville et de maire de commune sont incompatibles. Le maire de la ville ou son délégué est le représentant de l'administration de la ville dans tous les conseils, commissions et organismes dans lesquels sa présentation est prévue par les lois et règlements en vigueur. Sous sa surveillance et sa responsabilité, le maire de la ville peut déléguer par arrêté une partie de ses compétences à un ou plusieurs de ses adjoints. En cas d'absence ou d'empêchement de ces derniers, le maire de la ville peut déléguer une partie de ses attributions à des membres du conseil. Tant qu'elles ne seront pas rapportées, ces délégations subsisteront. Par contre, lorsque le maire de la ville de qui elles émanent est décédé, suspendu, révoqué ou déclaré démissionnaire, ces délégations prennent fin sans être expressément rapportées. Aussi, en tant que chef des services administratifs et techniques de la ville, il nomme le secrétaire général de la ville, après avis consultatif du représentant de l'Etat parmi les agents et fonctionnaires de la hiérarchie A de la fonction publique, ou de niveau équivalent, dans des conditions précisées par décret. A cet effet, le secrétaire général assiste aux réunions du bureau avec voix consultative. Le maire de la ville associe le maire de la commune à l'étude des conditions générales de réalisation et à l'exécution des projets d'infrastructures et d'équipements prévus, en tout ou partie, dans les limites de la commune. Le maire de la commune en rend compte à la plus proche séance du conseil municipal. Le maire de la commune doit aussi informer au maire de la ville des investissements entrepris dans le cadre des compétences de la commune. Le secrétaire général assiste aux réunions du bureau, avec voix consultative. Le maire met fin à ses fonctions dans les mêmes formes ${ }^{13}$.

A ces innovations institutionnelles, s'ajoutent les innovations fonctionnelles.

\section{Sur le plan fonctionnel}

- Nouvelle collectivité locale, le département bénéficie de compétences étendues avec l'avènement du CGCL. Ce vaste domaine de compétences dont bénéficie le département est surtout noté dans le cadre des compétences transférées. Le département exerce ainsi les compétences qui étaient dévolues jusque-là à la région, ancienne collectivité locale. Il s'agit fondamentalement des compétences transférées aux collectivités locales dans les secteurs suivants : domaine; environnement et gestion des ressources naturelles; santé, population et action sociale; jeunesse, sports et loisirs; culture; éducation; planifica-

13 Article 186 du code général des collectivités locales. 
tion; aménagement du territoire; urbanisme et habitat. Aussi, notons que pour l'exercice de certaines compétences dont l'avis du conseil rural était recueilli, cet avis est dorénavant du ressort du conseil municipal. D'un autre côté, en plus des compétences auparavant exercées par la région, le département se voit octroyer certaines compétences de la communauté rurale d'alors et de la commune d'une part, et, d'autre part, on assiste à la naissance de toutes nouvelles compétences. Somme toute, le département s'est vu octroyer un large domaine de compétences.

- Le CGCL confère à la commune également des compétences très étendues. En effet, même si certaines compétences de la commune ont été supprimées, il n'en demeure pas moins que l'essentiel de ses compétences sont maintenues mais également la commune a droit à de nouvelles compétences transférées. Il s'agit, d'une part, pour l'essentiel de compétences transférées qui étaient exercées par la communauté rurale et la région, et d'autre part il est question de l'apparition de nouvelles compétences transférées.

- Quant à la ville, ses compétences sont multiples et variées. Le législateur les classe en trois catégories : les compétences générales, les compétences spécifiques et les compétences transférées (voir CGCL).

Par ailleurs, des innovations fonctionnelles précitées, s'ajoutent celles notées sur le plan financier ainsi que la revalorisation de la participation citoyenne.

- En effet, si avec l'ancien code, les communes d'arrondissement devenues communes dépendaient partiellement du budget de la ville pour fonctionner, avec l'avènement du CG$\mathrm{CL}$, ces nouvelles communes deviennent autonomes vis-à-vis de la ville. Il en est ainsi parce qu'il ressort de l'article 193 du CGCL que les recettes ordinaires des collectivités locales proviennent du produit des recettes fiscales, de l'exploitation du domaine et des services locaux, des ristournes accordées par l'Etat ou d'autres collectivités publiques sur le montant des impôts et taxes recouvrés à leur profit, et de la répartition annuelle du fonds de dotation de la décentralisation. Il n'est fait aucunement mention dans le CGCL que les communes d'arrondissement devenues communes bénéficient de subventions de la part de la ville comme en attestait d'ailleurs l'article 23 du code des collectivités locales de 1996, qui dispose que les recettes de fonctionnement de la commune d'arrondissement proviennent des recettes fiscales limitativement énumérées, des ressources issues de subventions accordées par la ville.

En outre, l'article 29 de l'ancien code mettait en exergue le caractère obligatoire de cette dotation en stipulant que « est obligatoire la dotation que la ville verse chaque année aux communes d'arrondissement situées sur son territoire ». C'est dire que le nouveau code des collectivités locales a mis fin à la dépendance financière des communes d'arrondissement (devenues communes) vis-à-vis de la ville. Les nouvelles communes sont devenues autonomes vis-à-vis de la ville. Par contre, cette dernière dépend désormais partiellement des communes pour fonctionner. Autrement-dit, les communes sont appelées à participer financièrement au fonctionnement de la ville en contribuant au budget de celle-ci. Ceci est mis en exergue par l'article 185 (4) du CGCL. En effet, le législateur, en énumérant les recettes de la ville au niveau de l'article 185 dudit code, a mentionné qu'en dehors des recettes fis- 
cales, des revenus du patrimoine de la ville, des contributions du fonds de dotation de la décentralisation et toutes autres ressources dont la perception est autorisée par les lois et règlements, la ville bénéficie tout aussi des contributions des communes à son budget.

De plus, les anciennes communautés rurales devenues communes, qui avaient jusque-là comme ressources propres la taxe rurale, verront leurs ressources fiscales se multiplier. En conséquence, les nouvelles communes bénéficient avec la nouvelle réforme de la diversité des recettes de fonctionnement octroyées jusque-là aux communes. Aussi, en dehors des recettes de fonctionnement, les nouvelles communes ont également droit aux recettes d'investissement dont bénéficient les collectivités locales.

- D’un autre côté, une certaine innovation est également notée dans le cadre du concours financier de l'Etat. Si, dans le cadre de l'ancien CCL, le fonds de dotation était réparti en trois composantes (une part pour compenser les compétences transférées, une autre pour les services déconcentrés de l'Etat qui concourent au fonctionnement des CL et la dernière part pour le fonctionnement des services de la région), le législateur du nouveau code réduit ce fonds à deux volets. Pour ce qui est du FECL, il n'y a pas eu d'innovation. Le législateur maintient les dispositions de l'ancien code.

Par ailleurs, si des innovations sont notées sur le plan financier, une autre innovation apportée par le CGCL retiendra également notre attention. Il s'agit de l'élévation de la participation citoyenne au rang de principe au même titre que la libre administration.

- Jusqu'en 2013, la participation citoyenne était évoquée dans le cadre des dispositions générales du code des collectivités locales. Avec la nouvelle réforme, la participation citoyenne a été élevée au rang de principe au même titre que le principe de la libre administration des collectivités locales dans un souci de donner plus d'ampleur à l'implication des citoyens dans la mise en œuvre des politiques locales. A cet effet, le CGCL met en évidence deux types de participation citoyenne. Il s'agit d'un côté de la participation directe du citoyen dans la gestion des affaires locales qui se manifeste par le droit d'accès à l'information et la possibilité d'émettre des propositions. D'un autre côté, la participation du citoyen est indirecte dans la mesure où le citoyen intervient par l'institution d'un cadre de concertation dans chaque collectivité locale (voir articles 6 et 7 du CGCL).

En Somme, voilà évoquer de façon substantielle les innovations apportées par le CGCL. Cependant, une analyse approfondie des dispositions de cedit code révèle des limites considérables. C'est d'ailleurs tout l'intérêt d'aborder les lacunes du CGCL dans le cadre de la seconde partie avant de procéder à la formulation de quelques suggestions en vue d'améliorer ce code.

\section{II) Les limites des innovations du code général des collectivités du Sénégal}

La réforme effectuée par le législateur était nécessaire eu égard aux maux dont souffrent les collectivités locales sénégalaises. En d'autres termes, au regard des lacunes que présentaient l'ancien code des collectivités mais également relativement à l'appréciation faite de 
la pratique de la décentralisation, il était primordial voire indispensable de procéder à un changement à la politique de décentralisation sénégalaise. Donc, l'initiative prise par le gouvernement d'enclencher une nouvelle réforme des collectivités locales est à saluer. Cependant, grande fut la déception à la lecture des dispositions de cette dite réforme, qui, non seulement se trouvent être une transposition de l'essentiel des dispositions de l'ancien code, mais les réels problèmes auxquels sont confrontées les entités locales décentralisées n'ont pas été pris en compte. De ce fait, il serait intéressant d'évoquer dans le cadre de cette seconde partie les lacunes du CGCL(A) avant d'essayer de proposer quelques solutions dans un souci d'améliorer cedit code(B).

\section{A) Les lacunes du code général des collectivités locales}

Les lacunes décelées dans le code général des collectivités locales peuvent essentiellement être situées à deux niveaux à savoir sur le plan juridico-fonctionnel mais également sur le plan financier.

- Sur le plan juridique, nous avons essentiellement constaté la transposition des dispositions de l'ancien code des collectivités locales mais aussi la lenteur quant à l'élaboration de tous les décrets d'application. En effet, il est dommage de constater que l'essentiel des dispositions élaborées dans cedit code n'est qu'une transposition de l'ancien code des collectivités locales. Cette remarque apparaît à première vue dans le cadre des dispositions régissant le département comme nouvelle collectivité locale. Fondamentalement, à ce niveau, la réglementation relative à l'organisation administrative et financière du département n'est qu'une transposition pure et simple de celle de la région, ancienne collectivité locale. Donc, l'on est tenté de se poser la question de savoir : fallait-il supprimer la région? Cette interrogation s'avère pertinente si l'on sait que la région a fait l'objet de critiques du fait notamment de l'absence de fiscalité propre. Elle dépendait essentiellement du fonds de dotation de la décentralisation que lui octroyait l'Etat en vue d'assurer son fonctionnement. Alors, pourquoi avoir repris les mêmes dispositions pour régir le département? Ne fallait-il pas essayer d'apporter une solution aux problèmes dont souffrait cette collectivité au lieu de procéder à sa suppression pure et simple? Par ailleurs, relativement à la communalisation intégrale, cette innovation pourrait être une des plus importantes si l'on avait pris en compte la spécificité des nouvelles communes plus particulièrement des communautés rurales devenues communes. Cela veut dire quoi? Cela veut dire tout simplement que la ruralité qui caractérise les collectivités sénégalaises devrait être mise en exergue en leur donnant la dénomination de « communes rurales » à la place de « communes » tout court. Il en est ainsi parce que la commune, au vrai sens du terme, renferme des particularités permettant de la distinguer des autres ordres de collectivités locales. A cet effet, il est clairement établi au niveau de l'article 73 du CGCL que « ne peuvent être constituées en communes que les localités ayant un développement suffisant pour pouvoir disposer des ressources propres nécessaires à l'équilibre de leur budget ». A la lecture de cette disposition, il semble que certaines 
communautés rurales érigées en communes sont loin de remplir les conditions posées à l'article précité si l'on sait que la majorité de ces collectivités sont caractérisées par leur pauvreté. Donc, à ce niveau l'on se pose également la question de savoir s'il était judicieux de supprimer les communautés rurales? On ne voit vraiment pas la pertinence de modifier la dénomination d'un aspect alors que cet aspect en tant que tel n'a pas changé. Encore une fois et comme pour la plupart du temps, le législateur sénégalais s'est limité à copier le modèle français sans tenir compte des réalités du pays.

Par ailleurs, si les lacunes du CGCL se mesurent d'une part sur le plan juridique à travers la transposition pour l'essentiel du CCL, elles se manifestent d'autre part, par rapport à la lenteur de l'élaboration de tous les décrets d'application.

- La loi 2013-10 du 28 décembre 2013 portant code général des collectivités locales à elle seule ne suffit pas pour appréhender la réforme. Bien, évidemment elle doit s'accompagner de dispositions réglementaires qui doivent à cet effet préciser les modalités d'application de la loi. Or, depuis son adoption jusqu'à ce jour, l'ensemble des décrets d'application ne sont pas encore élaborés. Les seuls décrets d'application disponibles sont : le décret $n^{\circ}$ 2014-1222 fixant le statut du secrétaire municipal, le décret n²014-1223 fixant les taux maxima des indemnités et frais alloués aux membres du bureau du conseil départemental, aux conseillers et aux membres de la délégation spéciale du département, le décret n²014-1224 fixant le statut du secrétaire général de département ou de ville, le décret $n^{\circ}$ 2014-1225 fixant les taux maxima des indemnités et frais alloués aux membres du bureau municipal, et aux membres de la délégation spéciale de la ville et de la commune. Cette carence de dispositions réglementaires est vraiment déplorable. Par exemple en ce qui concerne l'état civil, l'élaboration des décrets d'application nous permettrait de savoir comment le gouvernement entend régir la gestion de l'état civil au sein des communautés rurales devenues communes, qui étaient jusque-là des centres secondaires ne disposant pas de locaux et de personnel compétent. Aussi, les aspects importants de la réforme notamment pour ce qui est du transfert des compétences et des dispositions relatives au financement des nouvelles collectivités locales mériteraient de réels éclaircissements. Donc, il urge d'élaborer les différents décrets d'application pour une meilleure application de la réforme

Sur le plan fonctionnel, il est noté une carence d'un dispositif pour la promotion de la qualité des ressources humaines, mais également des manquements quant aux modalités de transfert des compétences.

- Il est à signaler que le législateur du CGCL n'a pas pris en considération un aspect crucial entrant dans le cadre d'une réelle politique de décentralisation qui n'est rien d'autre que la promotion de la qualité des ressources humaines. L'on n'est pas sans savoir que nombreux sont les élus locaux qui ne comprennent pas les textes de la décentralisation. Dès lors, le législateur aurait dû prévoir des mesures allant dans le sens de l'amélioration de la qualité des acteurs locaux. Le niveau intellectuel de certains élus dans certaines localités s'avère faible. L'étude menée par une équipe de chercheurs révèle que « l'accession au pouvoir de beaucoup d'élus locaux est plus l'émanation de l'autorité centrale et 
des partis dominants, à partir de consignes de vote, que d'un choix populaire incarnant la confiance d'une communauté, en des personnes vertueuses et compétentes. De ce fait, une fois élu, l'exécutif local s'illustre par des abus de pouvoirs et une velléité de gouvernance solitaire et opaque $\mathrm{du}$ fait du déséquilibre des pouvoirs à l'intérieur des conseils $\rangle^{14}$. De ce fait, le clientélisme politique est à bannir dans une perspective d'efficacité de la gestion des collectivités locales. A cet effet, il serait important que le législateur élabore des dispositions allant dans le sens de la promotion de la qualité des élus locaux. Il est ainsi indispensable d'intensifier leurs capacités techniques et professionnelles pour une meilleure gestion des affaires locales. Par ailleurs, si la qualité des ressources humaines dans certaines entités locales laisse à désirer, des manquements sont également notés au niveau des modalités du transfert de compétences.

- Pour ce qui est des compétences transférées, mis à part quelques nouvelles compétences qui ont été introduites, le CGCL n'a fait que maintenir les neuf domaines de compétences créées par la loi 96-07 du 22 mars 1996. Aussi, le législateur sénégalais a pris comme choix de transférer les mêmes domaines de compétences à toutes les collectivités locales. Ainsi, le transfert des compétences de l'Etat aux collectivités locales s'est effectué sans une réelle prise en compte de la spécificité de l'entité locale. C'est dire que le législateur n'a pas procédé à la détermination du niveau le plus pertinent pour l'exercice d'une compétence bien définie. Il n'a pas opté pour le système qui consiste à transférer des compétences à chaque collectivité en fonction de ses besoins et de ses capacités.

D'un autre côté, si des manquements sont constatés au niveau fonctionnel, il apparaît également une insuffisance notoire des ressources financières des entités locales.

- L'un des problèmes majeurs de la politique de décentralisation au Sénégal est le défaut de mobilisation de fonds nécessaires en vue de la bonne gestion des affaires locales. Il en est ainsi parce que les ressources financières des collectivités locales sénégalaises sont très insuffisantes par rapport à leurs besoins. Insuffisance due essentiellement d'un côté à une fiscalité locale déficiente mais aussi à un défaut de recouvrement des impôts locaux, et, d'un autre côté, les fonds octroyés par l'Etat (FDD et FECL) sont dérisoires.

- En effet, tout d'abord, il est malheureux de constater que le département ne dispose pas d'une fiscalité propre. Il n'a pas été défini dans le cadre du code général des collectivités locales une fiscalité propre pour le département. Le législateur s'est contenté de procéder à une transposition des dispositions de l'ancien code des collectivités locales. En effet, les ressources financières qui étaient auparavant allouées à la région demeurent les mêmes pour le département (article 194 du CGCL). Le département ne dispose pour l'heure d'aucune autonomie financière. Ainsi, le département fonctionne essentiellement

14 Dr MAlam MOUSSA, Laouali et Dr ILBOUDO, Ernest K., Décentralisation en Afrique de l'Ouest et du Centre : apprendre des expériences locales et intersectorielles, Education, Eau, Santé, EDUCI, 2012. 
avec les ressources attribuées par l'Etat à travers le fonds de dotation, contrairement à la commune qui bénéficie à la fois des ressources fiscales et du concours de l'Etat.

Par ailleurs, l'autre aspect qui concourt à rendre dérisoire la fiscalité locale est l'existence d'une multitude d'exonérations. Le professeur Moussa ZAKI dans son article intitulé « les entraves à l'autonomie financière des collectivités locales au Sénégal ${ }^{15}$ publié en juin 2014, définit les exonérations comme étant des mesures ayant pour objet de soustraire partiellement ou totalement une matière du champ d'application de l'impôt; temporaires ou permanentes, elles sont accordées par la loi. En conséquence, ces exonérations entrainent de nombreuses pertes de recettes dans la mesure où il n'existe pas de mécanismes de compensation à ces dites exemptions. A cela s'ajoute l'impossibilité pour les collectivités locales de fixer le taux de l'impôt encore moins d'aménager l'assiette.

- Sous un autre registre, le législateur aurait dû élaborer des dispositions allant dans le sens de la résolution définitive de la problématique du recouvrement des impôts locaux. Il en est ainsi parce que le recouvrement des impôts locaux demeure toujours problématique. Le rapport national de la gouvernance au Sénégal ${ }^{16}$ publié en janvier 2013, révèle que dans la commune de Nioro seuls $5 \%$ de la population paie la taxe municipale. En fait, la majorité de la population n'a pas conscience de l'importance de l'impôt dans la mise en œuvre de projets d'envergure dans l'espace communal. De plus, la mairie n'a pas mis en place une stratégie de communication devant permettre aux populations de prendre en charge la plénitude de leurs responsabilités dans la conduite des affaires publiques.

Sous un autre angle, le rapport général sur les assises nationales de la décentralisation précité, évoque des causes sociopolitiques. En effet, il ressort de cedit rapport que les contribuables manifestent de plus en plus de réticence à s'acquitter de l'impôt. Ils justifient leur comportement par leur insatisfaction par rapport aux services obtenus de la collectivité locale, l'absence de transparence au niveau de la gestion du produit des impôts et, surtout, par leur non-implication dans la prise de décision concernant le développement de leur localité $^{17}$.

De ce fait, face à cette réticence des populations, le recouvrement des impôts locaux s'avère problématique engendrant à cet effet d'énormes incidences négatives sur le plan financier. Ainsi, malgré la panoplie de ressources budgétaires prévues par la loi, la pratique est tout autre quant à leur perception. Ceci étant dû à l'absence d'activités économiques mais également la pauvreté qui caractérisent certaines localités. C'est ce qui pousse d'ailleurs les entités locales à dépendre pour l'essentiel du concours financier de l'Etat et de l'aide extérieure pour assurer tant soit peu le fonctionnement de la collectivité. Ceci étant, le législateur aurait dû prendre en compte ce facteur (c'est-à-dire la problématique du re-

15 http://afrilex.u-bordeaux4.fr/sites/afrilex/IMG/pdf/ZAKI_article_finances_loc2.pdf.

16 http://www.sn.undp.org/content/dam/senegal/docs/gouvernance/undp-sn-Rapport National gouvernance jan 2013.pdf.

17 Rapport des assises sur la décentralisation au Sénégal, 2007, in https://www.google.sn/-. 
couvrement des impôts locaux) qui bloque d'une manière ou d'une autre le bon fonctionnement de la gestion des affaires locales.

Par ailleurs, en plus de l'insuffisance de ressources financières propres, les collectivités locales font face également à une faiblesse des fonds que leur octroie l'Etat.

- L'Etat a procédé au transfert de neuf domaines de compétences aux collectivités locales sans une réelle compensation financière de ces charges. Le taux prélevé sur le budget de l'Etat pour alimenter le FDD et le FECL demeure très minime par rapport aux compétences transférées. En effet, le fonds de dotation, principal mécanisme de financement des charges issues du transfert de compétences, renferme deux insuffisances essentielles à savoir le caractère infime du taux de la TVA perçu sur le budget de l'Etat (3.5\%) mais également le manque de pertinence des critères de répartition de ce fonds. Le montant du taux prélevé sur le budget de l'Etat (3,5\%) est très médiocre par rapport aux compétences transférées. De plus, la pratique a également révélé que les collectivités locales reçoivent tardivement ce fonds.

- Tout comme le FDD, le FECL demeure également très insuffisant. Le taux alloué à ce fonds est très en deçà par rapport aux besoins des collectivités locales en matière d'investissement. Aussi, au regard du nombre sans cesse grandissant des collectivités locales sénégalaises, le montant de $2 \%$ de la TVA allouée pour le FECL se révèle largement insuffisant. En effet, l'investissement demeure très important dans la recherche de la croissance économique dans la mesure où il contribue à la création d'infrastructures et favorise la production de services publics. De cette façon, il nécessite la mobilisation de ressources financières suffisantes.

Mais tel n'est pas le cas dans les entités locales sénégalaises. En conséquence, le recours au financement extérieur devient nécessaire. Or, ce mode de financement actuel, avec notamment l'appui des partenaires extérieurs, n'est pas durable eu égard aux difficultés que rencontrent ceux-ci, notamment avec la crise économique qui est toujours d'actualité.

Somme toute, relativement aux limites décelées dans les dispositions du nouveau code des collectivités locales du Sénégal, il conviendrait de formuler quelques recommandations allant dans le sens de l'amélioration de cedit code.

B) La nécessaire amélioration du code général des collectivités locales

Une réforme de la politique de décentralisation au Sénégal était attendue eu égard aux nombreuses difficultés auxquelles sont confrontées les collectivités locales. Les autorités en place l'ont comprise et ont enclenché une nouvelle réforme connue sous le nom de l'acte 3 de la politique de décentralisation au Sénégal. Cependant, comme pour la plupart des réformes, celle-ci a été pour l'essentiel d'ordre institutionnel. Des solutions n'ont pas été apportées aux véritables problèmes (à savoir notamment le défaut de ressources financières suffisantes et la qualité des ressources humaines,...) rencontrés au sein des entités locales. Donc, le CGCL tel que élaboré n'est pas pertinent. Les dispositions ne répondent pas aux 
besoins réels de décentralisation du pays. C'est tout l'intérêt de formuler quelques suggestions en vue de l'amélioration du CGCL. En conséquence, des recommandations seront exposées d'une part sur le plan juridico-fonctionnel et d'autre part, sur le plan financier.

- Sur le plan juridique, certes le législateur du CGCL a procédé à la revalorisation de la participation citoyenne mais nous estimons que les dispositions peuvent être améliorées pour une meilleure et effective participation. A cet effet, le législateur doit envisager des mécanismes efficaces de contrôle des citoyens sur la gestion des collectivités locales dont ils sont membres. En quoi faisant? Il faudra veiller au strict respect du droit à l'information, notamment le droit d'accès aux documents administratifs. Pour ce faire, la mise en place effective d'une commission d'accès aux documents administratifs nous paraît nécessaire dans le but de veiller au droit reconnu au citoyen. Dans cette même perspective, il ressort du rapport national de la gouvernance au Sénégal précité, qu'une décentralisation qui donne effectivement le pouvoir aux collectivités locales serait également un gage de démocratie fondée sur une implication forte du citoyen dans la vie politique. Aussi, la gestion des affaires publiques, au plus près de la population, offre de grands avantages à savoir une meilleure connaissance des attentes, une possibilité de dialogue direct, plus de flexibilité dans la satisfaction des besoins, l'exercice du pouvoir de contrôle des citoyens sur les responsables ${ }^{18}$.

Par ailleurs, le législateur pourrait également élaborer des dispositions qui donneraient la possibilité aux citoyens de procéder à la révocation des autorités locales avant la tenue des élections si jamais les élus locaux ne remplissaient pas convenablement leurs missions. C'est dire que tout citoyen serait apte et prompt à contrôler la gestion des affaires publiques locales et surtout prêt à sanctionner, par tous les moyens légaux disponibles, les gestionnaires des affaires publiques. La citoyenneté n'est donc plus à construire mais plutôt à consolider ${ }^{19}$.

- D’un autre côté, au regard du contrôle exercé sur les actes des autorités locales, il apparait au niveau des dispositions du code que le contrôle a priori est marqué par une certaine lourdeur du fait de la forte présence du représentant de l'Etat. Ce dernier détient à la fois un pouvoir d'approbation préalable sur les actes des collectivités locales, un pouvoir de suspension et un pouvoir de substitution. A ce niveau, nous nous accorderons avec le professeur Ibrahima Diallo qui, dans son ouvrage intitulé « le droit des collectivités locales $»^{20}$, estime que « le contrôle a priori s'exerce concrètement sur un champ d'application très large et il s'exerce par nature de manière rigoureuse ». En effet, le pouvoir d'approbation et le pouvoir de suspension conféré au représentant de l'Etat occasionne un blocage dans l'exécution des actes locaux en ce sens que l'exercice de ces pouvoirs requiert le respect d'une procédure pouvant être long au regard des délais impartis, alors que les collectivités locales doivent pouvoir disposer des ressources finan-

18 Rapport national de la gouvernance au Sénégal, page 36.

19 Raogo Antoine Sawadogo, l'Etat africain face à la décentralisation, Editions Karthala, 2001.

20 Ibrahima DIALLO, Le Droit des Collectivités Locales au Sénégal, L’harmattan, page 164, 2007. 
cières à temps pour assurer leur fonctionnement. Les prérogatives ainsi conférées au représentant de l'Etat sont très étendues. Fort de ce constat, nous estimons que le contrôle exercé par le représentant de l'Etat doit être allégé. Ainsi, le législateur doit aller dans le sens de l'assouplissement du contrôle exercé par le représentant de l'Etat sur les actes locaux, qui semble remettre en cause la libre administration des collectivités locales.

Par ailleurs, sur le plan fonctionnel, il serait également judicieux d'envisager une réelle amélioration de la qualité des ressources humaines mais aussi de revoir les modalités de répartition des compétences.

- Il en est ainsi parce que l'efficacité d'une décentralisation dépend en partie de la conscience qu'ont les différents acteurs de leurs responsabilités mais également de leurs capacités à impulser le développement local à travers des actions pertinentes. Dans un souci de promouvoir une bonne politique de la décentralisation, il est primordial que la capacité des élus locaux soit renforcée. A notre avis, le législateur doit aller dans le sens de la redéfinition du statut de l'élu local. Il conviendra à cet effet de prendre des dispositions visant à mettre fin au clientélisme politique. Il serait à cet effet pertinent d'autoriser les candidatures indépendantes afin de favoriser les plus compétents et les plus méritants et d'instaurer à cet effet la confiance qui doit prévaloir entre élus locaux et citoyens locaux. Dans le rapport général des assises national de la décentralisation précité, il est même proposé l'élection des exécutifs locaux au suffrage universel. En outre, faire en sorte que les élus locaux maitrisent bien les dispositions légales et réglementaires régissant l'organisation et le fonctionnement des collectivités locales afin d'assurer une bonne gestion de leurs compétences; qu'ils sachent également leurs droits et obligations et soient de plus soumis à une obligation de résultats.

Donc, les critères de choix des élus locaux doivent être revus. A cet effet, pour renforcer la transparence et l'efficacité dans la gestion des affaires locales, ils (les élus locaux) doivent dresser chaque année un bilan au terme de leur gestion qui sera suivi d'une sanction de la part des populations. Aussi, il faut que les acteurs locaux soient aptes à identifier les besoins prioritaires des populations. Selon le rapport national de la gouvernance au Sénégal cité un peu plus haut, " l'instruction des élus même si elle ne conduit pas forcément à de bonnes performances dans la gestion, est jugé très utile. Elle rend possible la lecture du contenu des documents d'état civil avant signature, la compréhension plus rapide de la réglementation relative à la décentralisation, la maîtrise des procédures budgétaires ", entre autres.

Par ailleurs, le défaut d'information constitue une contrainte à l'implication des populations au processus de prise de décision. Les élus locaux doivent ainsi avoir la capacité de mobiliser les autres acteurs de la décentralisation dans un souci de coordonner leurs actions afin de promouvoir la satisfaction de l'intérêt général des collectivités. Ainsi, il est important de renforcer et de rendre dynamique la collaboration entre les acteurs locaux. De plus, les élus locaux doivent être en mesure d'élaborer des programmes et des projets de développement cohérents et adaptés tout en tenant compte des spécificités des entités 
locales. En somme, la promotion de la qualité des ressources humaines est incontournable pour la mise en œuvre effective de la décentralisation.

En dehors de cet aspect crucial, il est tout aussi important de procéder à la définition de nouveaux mécanismes dans le cadre de la répartition des compétences transférées.

- Il s'agira à ce niveau d'améliorer le processus du transfert et de la mise en œuvre des compétences en fonction des besoins réels de la collectivité concernée. A notre avis, procéder au transfert des mêmes compétences à toutes les collectivités n'est pas pertinent. Le transfert d'une compétence à une collectivité locale doit être subordonné aux besoins réels de celle-ci. Pour ce faire, une étude de terrain s'impose afin de pouvoir déterminer ces dits besoins. Il est ainsi question de connaître les atouts et les potentialités de chaque collectivité locale en vue de les exploiter de façon efficiente. Ce qui permettrait d'ailleurs d'exercer la compétence au niveau qui convient le mieux. Ainsi, la prise en compte de la situation et de la dynamique locale s'avère indispensable dans le processus de transfert de compétences aux collectivités locales. L'efficacité même de ce transfert de compétences passe nécessairement par la prise en compte de la spécificité de la localité en question.

Chaque collectivité renferme ses propres réalités. De ce fait, les besoins et les préoccupations diffèrent en fonction de la localité en cause. Il s'agira à cet effet de procéder à l'identification des problèmes cruciaux auxquels sont confrontées au jour le jour les collectivités locales pour ensuite responsabiliser les acteurs locaux par le biais du transfert de compétences. Du moment où tous les problèmes et besoins ne sont pas partagés, les compétences ne doivent pas non plus être partagées. Donc, il conviendra de prendre des dispositions au regard des réalités et spécificités socio-économiques des entités locales. Par exemple, si certaines localités sont confrontées à des problèmes d'accès à l'eau ou à l'éclairage public, d'autres, par contre, souffrent de l'état dégradé des routes. Partant, la prise en compte de la spécificité locale dans le processus de transfert de compétences est nécessaire.

- Sur le plan financier, une mobilisation effective des ressources financières s'impose pour l'affirmation d'une décentralisation effective. Pour ce faire, une revalorisation des ressources propres des collectivités locales doit être envisagée, aussi l'Etat doit revoir considérablement à la hausse ses concours financiers.

La revalorisation des ressources propres des collectivités locales suppose non seulement de procéder à la modernisation de la fiscalité locale mais aussi de définir des mécanismes pour assurer le recouvrement effectif des impôts locaux.

- En effet, la structure de la fiscalité locale occasionne dans une certaine mesure une inégalité au sein des collectivités locales dans la mesure où on est en face de collectivités « riches » d'une part, et, de collectivités « pauvres », d'autre part. Il en est ainsi parce que les impôts créés par le législateur (la patente, la contribution foncière, la licence, l'impôt sur l'immobilier) ne sont productifs que pour les grands centres. Sur ce, le législateur sénégalais pourrait aller dans le même sens que son homologue burkinabé dans son processus de modernisation de la fiscalité locale. 
Il ressort de l'ouvrage de Raogo Antoine SAWADOGO précité que les communes du Burkina Faso ont très vite mis en œuvre des mesures multiples et créatives pour accroître les recettes, les rémunérations pour services rendus et taxes diverses. Elles ont utilisé une véritable panoplie de moyens : reprise de la perception des taxes tombées en désuétude, relèvement de taux qui n'avaient pas été modifiés depuis plusieurs années, création de nouvelles taxes et amendes (la commune de Dori a institué des impôts, des taxes et amendes pour tous ceux qui ne respecteraient pas les règles d'hygiène publique), amélioration des méthodes de recouvrement, sensibilisation ${ }^{21}$. A la suite de cette initiative, il a été constaté que les résultats ont été significatifs. En effet, en 1995, le budget des communes s'élevait à 2,5milliards de franc CFA environ, et en 1999, à 13milliards.

Dans cette même perspective, nous aborderons dans le même sens que Pape Mor NDIAYE, qui dans son article intitulé « De la décentralisation fiscale $»^{22}$ asserte qu'un toilettage de la législation est indispensable. Selon lui, il s'agira juste de conserver les seuls impôts, taxes et redevances susceptibles de rendement minimal.

Aussi, l'octroi d'un pouvoir fiscal local aux élus locaux doit également être envisagé. Ceci est même rendu nécessaire par la directive $n^{\circ} 1 / 2011 / C M / U E M O A$ portant régime financier des collectivités territoriales. En effet, aux termes de l'article 16 de cette directive, le conseil de collectivité territoriale, par sa délibération fixe le taux des impôts et taxes locaux dans la limite du plafond fixé par la loi. Ainsi, les collectivités locales doivent avoir la possibilité de créer des impôts locaux et d'en fixer également le taux dans la mesure où chaque entité locale renferme sa particularité.

- A cela s'ajoute la définition de mécanismes pour parer aux obstacles liés au recouvrement des impôts locaux. Le niveau de recouvrement des impôts locaux demeure très faible. Les impôts locaux sont variés mais peu rentables et certains impôts et taxes ne sont pas recouvrés. De ce fait, au regard des contraintes décelées dans le cadre du recouvrement des impôts locaux, il est essentiel de définir des mécanismes allant dans le sens de l'amélioration des méthodes de recouvrement de ces dits impôts. A notre avis, la sensibilisation du citoyen sur l'importance du recouvrement de l'impôt demeure primordiale. Le sensibiliser revient à le conscientiser sur son implication effective dans le processus de développement local, du développement de la collectivité dont il est membre; lui faire comprendre que s'acquitter de l'impôt est un devoir citoyen et qu'en retour, les élus locaux tâcheront de subvenir aux besoins de la population. Dans cette même perspective, Raogo Antoine SAWADOGO estime que l'existence d'une conscience participative et contributive constitue un atout incontournable dans l'émergence de la nouvelle citoyenneté et dans la recherche de la souveraineté fiscale des collectivités locales. Cependant, il faudrait trouver ou veiller à intégrer dans les programmes de développement

21 Raogo Antoine SAWADOGO, l'Etat africain face à la décentralisation, Editions Karthala, 2001.

$22 \mathrm{http} / /$ www.amcod.info/fr/publications/fiscalite_transfert/senegal/110711_Intervention_Pape_Mor _Ndiaye_DECENTRALISATION_FISCALE CADDDEL.pdf. 
local des « affaires » socialement finançables qui correspondent aux aspirations des populations et qui sont collectivement légitimées ${ }^{23}$.

C'est dire que la mobilisation des ressources doit être suivie de la mise en place des infrastructures locales et des services publics locaux dont les populations ont besoin. C'est entre autres l'une des techniques qui devrait encourager le citoyen à s'acquitter de l'impôt afin de pouvoir contribuer au développement de sa collectivité. Par conséquent, ceci faciliterait sans doute le recouvrement des impôts locaux.

Sous un autre registre, l'Etat doit également songer à octroyer un financement très conséquent en vue de la compensation des charges transférées. Pour ce faire, il est nécessaire d'accroître les FDD et le FECL et de procéder à la redéfinition des critères de répartition de ces fonds.

Afin de garantir l'efficacité de la gestion des affaires locales, il est impératif de mettre à la disposition des collectivités locales les moyens et les ressources nécessaires à la mise en œuvre des compétences transférées en respectant le principe de la concomitance. En effet, il est inconcevable que le pouvoir central charge les collectivités locales sans pour autant qu'il y ait une compensation financière couvrant ces charges. Comment concevoir que l'Etat puisse transférer une partie de ses compétences aux collectivités locales sans de véritables mesures d'accompagnement pour celles-ci? En d'autres termes, comment l'Etat peutil transférer des charges aux entités locales sans moyens financiers conséquents? C'est dire que l'appui financier de l'Etat est incontournable au regard des charges qu'il a transférées aux entités locales.

Ainsi, nous aborderons dans le même sens que les conclusions faites lors des assises de la décentralisation en 2007. En effet, il est fait mention dans le rapport que l'appui financier de l'Etat devrait être renforcé pour permettre aux collectivités locales d'assurer un développement local performant. De ce fait, le FDD et le FECL doivent être substantiellement rehaussés. La part de la TVA affectée aux collectivités locales équivaut à 5.5\%. Une augmentation de ce taux à hauteur de 15 à $20 \%$ doit être envisagée. Le Maroc par exemple confère à ses collectivités décentralisées, un taux de la TVA égale à 30\%. En effet, il ressort de l'exposé $^{24}$ mené sur la situation financière des collectivités locales en Afrique en 2009 qu'en Maroc, depuis 1988, 30\% du produit de la TVA sont transférés aux collectivités locales, représentant en moyenne près de la moitié des ressources locales. Pourquoi, le Sénégal n'irait pas dans le même sens que l'Etat marocain. En France, la dotation avoisine les $20 \%$ du budget de l'Etat. Le Sénégal pourrait aller dans le même sens.

En définitive, le financement de l'Etat au profit des collectivités locales doit nécessairement être rehaussé.

De plus, les critères de répartition du FDD et du FECL doivent être également redéfinis.

23 Raogo Antoine SAWADOGO, l'Etat africain face à la décentralisation, Editions Karthala, 2001.

24 Cheikhou DIOP, « Situation des Finances des Collectivités locales en Afrique/Caractéristiques des Collectivités locales en Afrique : état des lieux », CIFAL Ouagadougou, Juin 2009. 
A ce niveau, il urge d'adopter des critères rationnels de répartition du FDD et du FECL entre les collectivités mais aussi de prôner le principe de la transparence dans la répartition. En outre, ces fonds doit être alloué en prônant également l'équité dans la répartition entre collectivités locales. Ceci étant, la part du FDD réservée pour la compensation des compétences transférées doit être affectée à chaque collectivité en tenant compte de ses priorités. Autrement-dit, il s'agira d'allouer à chaque entité décentralisée en fonction de ses besoins prioritaires.

Aussi, ce fonds doit nécessairement tenir compte du niveau de pauvreté des collectivités locales. A ce niveau, nous estimons que les collectivités locales « riches » c'est-à-dire celles qui parviennent à mobiliser leurs ressources financières de façon considérable à travers un recouvrement effectif de leurs impôts et taxes locaux, ne doivent pas bénéficier de ce fonds. Il est question de privilégier les collectivités « pauvres ».

Par ailleurs, pour ce qui est du FECL, le critère de performance établi pour l'allocation $\mathrm{du}$ fonds de concours ordinaire peut être maintenu. Par contre, pour ce qui est du critère défini pour l'allocation du fonds de concours spécial, celui-ci pourrait être revu. Il conviendra à ce niveau d'apprécier de la pertinence du projet envisagé. Apprécier la pertinence du projet, reviendrait à analyser si le projet en cause apporterait un plus à la localité en terme de service public ou d'infrastructures. Dans cette même optique, le professeur Mayacine Diagne, dans son cours de finances locales estime que dans le cadre de l'attribution du fonds de concours spécial, devrait être privilégié, les collectivités locales qui disposent d'un document de planification stratégique.

\section{Conclusion}

Au Sénégal, de l'indépendance jusqu'à nos jours, une panoplie de textes régissant la politique de décentralisation a été mise en place. La décentralisation est ainsi une volonté politique exprimée depuis l'indépendance. Conscientes de la mise en place d'une administration de proximité, les autorités sénégalaises se sont lancées dans un processus de désengorgement du pouvoir central mis en évidence par la politique de décentralisation. L'étude de ce sujet a permis non seulement d'exposer le contenu de la phase une de «l'acte 3 » de la décentralisation, mais a également permis de soulever les problèmes rencontrés dans le processus de la décentralisation qui n’ont pas été solutionnés par cette dite réforme. De par leurs organes et leurs missions, les collectivités locales se présentent comme des actrices incontournables pour une réelle promotion du développement au sein des entités locales. Si depuis 1996 des avancées notoires ont été enregistrées en terme de responsabilisation des entités locales dans la conduite des affaires locales, il n'en demeure pas moins que des carences et des obstacles sont à noter dans la satisfaction des besoins sociaux des populations. Partant, la carence en ressources humaines et financières constitue un sérieux handicap pour le plein essor de la politique de décentralisation au Sénégal. Une prochaine réforme devrait pouvoir apporter des solutions définitives à ces maux dont souffre l'administration locale sénégalaise. En effet, il urge pour l'Etat de prendre des mesures adéquates par rapport aux 
attentes des populations pour une bonne gouvernance locale. Il devrait en être ainsi parce que si toutes les collectivités locales se développent, c'est tout le Sénégal qui émerge. Ceci étant dit, il convient de souligner qu'il y'a encore beaucoup à faire au niveau des textes mais également en ce qui concerne la distribution des ressources (humaines et financières) pour permettre aux entités décentralisées de répondre aux besoins des populations. La question fondamentale demeure le problème de l'autonomie financière mais également celui de la capacité des élus locaux à faire face aux compétences qui leur sont dévolues. Nous nous accorderons avec Djibril Diop qui estime qu'au-delà de la nécessité de développer et de transformer le cadre institutionnel, le besoin le plus évident aujourd'hui est de faire en sorte que les collectivités locales aient les capacités nécessaires en matière d'infrastructures, de ressources humaines et surtout de moyens financiers pour réduire cette pauvreté galopante. Une décentralisation effective constitue un aspect qui favorise le processus de développement local. Ainsi, la collectivité locale se trouve être le cadre idéal pour l'impulsion du développement et la consolidation de la citoyenneté. Pour ce faire, il est essentiel que les dispositions législatives et réglementaires soient adoptées en fonction des réalités des collectivités locales. Une décentralisation réussie suppose la prise en compte de chaque spécificité locale pour l'impulsion d'un véritable développement local. Il est aussi fondamental de doter les collectivités locales d'un réel pouvoir de gestion propre à assurer une meilleure administration des affaires locales. En conséquence, le CGCL doit nécessairement être revu. 